\title{
C4d Glomerular Deposits and Disease Progression in Native Idiopathic Membranous Nephropathy
}

Vincenzo Sepe ${ }^{1^{\star}}$, Paolo Albrizio ${ }^{2}$, and Antonio Dal Canton ${ }^{1,2}$

${ }^{1}$ Unit of Nephrology, Dialysis, Transplantation, Fondazione I.R.C.C.S., Policlinico San Matteo, Italy

${ }^{2}$ Chair of Nephrology, University of Pavia, Viale Camillo Golgi, Italy

\begin{abstract}
Introduction: Since 1989 when Kusunoki et al. described C4d renal deposits in native idiopathic membranous nephropathy (nIMN) their role in disease progression has not been clarified yet. Recent studies have identified C4d glomerular staining as a marker of negative progression of renal function in a primary glomerular disease like IgA nephropathy. We have retrospectively analysed 15 consecutive formalin-fixed paraffin-embedded kidney biopsies from patients with nIMN (7F, 8M) performed in our Unit from October 1995 to February 2011.
\end{abstract}

Methods: Kidney sections were stained using polyclonal rabbit IgG anti-human C4d antibodies. Normal renal tissue was obtained from heart-beating braindead donors before kidney harvesting. Positive control biopsy was a humoral kidney rejection with intense $C 4 d$ staining. Data are expressed as $M \pm S D$.

Results: Ten (5F, 5M) of $15 \mathrm{nIMN}$ kidney biopsies showed global and diffuse C4d glomerular capillary staining

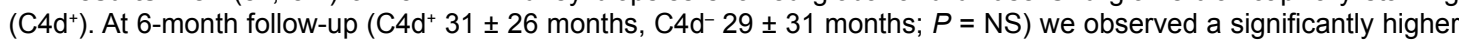
24-hour urinary protein excretion rate (UPr) in $\mathrm{C} \mathrm{d}^{+}\left(P=0.0051 \mathrm{vs.} \mathrm{C} 4 \mathrm{~d}^{-}\right)$, and a significantly lower MDRD eGFR $(P=$ 0.0337 vs. $\mathrm{C} \mathrm{d}^{-}$at diagnosis) when compared with data at disease presentation.

Conclusion: Our data suggest that C4d glomerular capillary deposits in nIMN with a follow-up longer than 6 months might be a negative prognostic factor for both UPr and eGFR. We are aware that our study has limitations like the relatively short term follow-up and the little number of biopsy analysed. Nevertheless, the association between increasing UPr, worsening of renal function and glomerular deposits of C4d in nIMN patients might deserve reporting and eventually confermation by further investigations.

Keywords: Chronic kidney disease; Complement; C4d; Idiopathic membranous nephropathy; Native kidney; Nephrotic syndrome; Proteinuria

\section{Background}

Increasing evidence from animal studies support the hypothesis that progression of renal failure in glomerular diseases is related by local complement activation [1]. Interstitial vascular C4d vascular deposits have been described in acute and chronic antibody-mediated rejection (ABMR) [2,3]. C4d is a surface-bound split product of inactive $\mathrm{C} 4 \mathrm{~b}$ obtained from classical or lectin complement pathways. To the date, receptor for $\mathrm{C} 4 \mathrm{~d}$ has not been identified and its biological function is unknown [4]. C4d positive staining $\left(\mathrm{C}^{2} \mathrm{~d}^{+}\right)$has also been reported in normal human kidneys [5], primary [6-8] and secondary $[9,10]$ nephropathies. The weak segmental $\mathrm{C} 4 \mathrm{~d}^{+}$glomerular staining observed in normal kidney biopsies possibly results by local complement activation involved in the physiological clearance of IgM-containing immune complexes [5]. A study on C4-deficient guinea-pig showed increased IgM glomerular deposits when compared to non C4ddeficient mammalian kidneys [11]. Human diseases studies suggest that $\mathrm{C}_{4} \mathrm{~d}^{+}$mesangial staining in patients with IgA nephropathy (IgAN) could identify individuals with a worse long-term prognosis [6]. In lupus nephritis (LN) glomerular $\mathrm{C} 4 \mathrm{~d}^{+}$staining has been associated with higher risk of developing thrombotic microangiopathy [12]. Interstitial peritubular $\mathrm{C}_{4} \mathrm{~d}^{+}$capillary deposits in LN appear to identify intense immunological disease activity [13].

Native idiopathic membranous nephropathy (nIMN) is an established antibody-mediated glomerular disease. A recent work has identified the M-type phospholipase A2 receptor (PLA2R), a $185-\mathrm{kD}$ glomerular glycoprotein as the target antigen in nIMN [14].

PLA2R is a podocyte protein expressed in normal glomeruli and
PLA2R antibodies are mainly of IgG4 isotype [14]. Experimental models have clarified that local injury is mediated by in situ immune complexes and complement activation [15]. However, IgG4 do not activate complement or very little only [16]. It may support the hypothesis that IgG4 act altering podocyte architecture and barrier function [14] suggesting that IgG subclasses other than IgG4 activate complement in nIMN binding PLA2R and/or other glomerular antigen(s).

Glomerular C4d deposits in nIMN sections has already been documented and often are used as positive control for C4d staining of renal grafts in order to support a diagnosis of ABMR in renal transplanted patients [17]. In absence of ABMR with stable renal function C4d deposits in graft glomerular capillaries appear to be associated with late ( $>6$ months) graft failure [18], but it is unknown the meaning of early and late outcome of nIMN with $\mathrm{C} \mathrm{d}^{+}$glomerular staining.

Aim of this retrospective study was to analyse changes of daily urine protein excretion and glomerular function in nIMN patients according to the presence of $\mathrm{C} 4 \mathrm{~d}$ deposits.

*Corresponding author: Vincenzo Sepe, Complex Structure Nephrology, Dialysis and Transplantation, Foundation I.R.C.C.S. Policlinico, San Matteo, Viale Camillo Golgi, 1927100 Pavia, Italy, Tel: +39-0382-50-2590; Fax: +39-0382-50-3666; E-mail:vincenzo.sepe@gmail.com; vsepe@libero.it

Received: September 16, 2014; Accepted: August 31, 2015; Published: September 06, 2015

Citation: Sepe V, Albrizio P, Canton AD (2015) C4d Glomerular Deposits and Disease Progression in Native Idiopathic Membranous Nephropathy. J Nephrol Ther 5: 212. doi:10.4172/2161-0959.1000212

Copyright: () 2015 Kuniyoshi Y. This is an open-access article distributed under the terms of the Creative Commons Attribution License, which permits unrestricted use, distribution, and reproduction in any medium, provided the original author and source are credited. 


\section{Materials and Methods}

From December 1995 to February 2011, 1207 native kidney biopsies have been performed in our Unit. Frozen and formalin-fixed paraffin-embedded kidney samples have been stored for all biopsies. Frozen sections have been tested for routine IF with FITC-conjugated rabbit anti-human IgG, IgA, IgM, C3, C4, fibrinogen, kappa and lambda light chains (Dako, Glostrup, Denmark). Paraffin sections are stained for histopathological evaluation with Harris hematoxylin and eosin, periodic acid-Schiff stain (PAS), methenamine-silver and Masson trichrome. Diagnosis and classification of nIMN glomerular lesions are carried out according to the classic staging by Ehrenreich and Churg as reported by Schawartz [19].

C4d routine staining for transplanted kidney biopsies was introduced in our Unit in May 2007. To date C4d staining has been performed in 10 kidneys from heart-beating braindead donors before transplantation and 97 transplanted renal allografts respectively.

C4d immunohistochemical detection was performed on formalin-fixed, paraffin-embedded kidney sections by indirect immunoperoxidase staining. Four $\mu \mathrm{m}$ thick sections cut from formalin-fixed paraffin-embedded renal tissue were deparaffinized, and endogenous peroxidase activity is blocked by 8 -min $1: 4,30 \%$ hydrogen peroxide dilution with distilled water. Antigen retrieval was obtained by 13 -min 1 bar pressure cooking at $250^{\circ} \mathrm{C}$. Primary polyclonal rabbit IgG anti-human C4d antibodies (Biomedica Group, Vienna, Austria) were applied at 1:30 dilution using Dako citomation solution (Dako, Glostrup, Denmark) [20] and incubated for 1 hour at $37^{\circ} \mathrm{C}$. Slides were then incubated with En Vision solution (Dako, Glostrup, Denmark) and staining was visualized by 3 , 3'diaminobenzidine. Immunoperoxidase reaction was blocked by distilled water and nuclear staining was obtained using Harris haematoxylin.

Negative control renal tissue was obtained from ten $(6 \mathrm{M}, 4 \mathrm{~F})$ previously mentioned heart-beating braindead donors ( $42 \pm 12$ year-

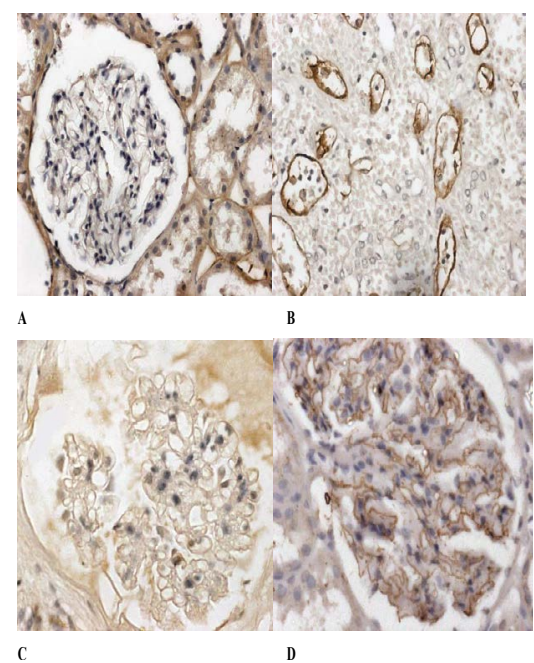

Figure 1: Immunoperoxidase staining of formalin-fixed, paraffin-embedded kidney sections using polyclonal rabbit IgG anti-human C4d antibodies.A, C4d negative control: Normal renal tissue obtained from heart-beating braindead donor before kidney retrieval; B, C4d positive control: Interstitial vascular $\mathrm{C} 4 \mathrm{~d}$ immunoperoxidase staining in antibody-related acute renal graft rejection; C, C4d negative immunoperoxidase staining of biopsy from nIMN (native Idiopathic Membranous Nephropathy) patient; D, C4d positive immunoperoxidase staining of biopsy from nIMN patient showing fine granular diffuse staining of glomerular capillary walls old) before kidney harvesting (Figure 1A). Positive control for C4d staining was renal graft sections from a kidney transplanted patient with $A B M R$ and intense C4d interstitial capillary staining (Figure 1B).

Data are expressed as mean \pm standard deviation. Statistical analysis was performed using T-test analysis. The null hypothesis was rejected when the $P$-value was less than 0.05 . All analyses were performed using the statistical package Stata 8.0 (Stata Corporation. College Station, Texas 77845 USA, 2003).

\section{Results}

Negative control biopsies showed glomerular, vascular and interstitial negative C4d immunohistochemistry (Figure 1A). All fifteen nIMN biopsies tested for C4d were retrieved from Caucasian patients $(7 \mathrm{M}, 8 \mathrm{~F})$ at diagnosis and before treatment. Mean age was $53.1 \pm 21.9$ years. Laboratory, histology and C4d immunohistochemistry findings are summarized in Table 1. Ten of five nIMN kidney biopsies showed glomerular capillary wall C4d deposits. At diagnosis no differences were observed between the two groups regarding age, sex, renal function, proteinuria, and disease stage (Table 1). Treatment induction with i.v. cyclophosphamide was administrated in 6 cases $\left(4 \mathrm{C}^{2} \mathrm{~d}^{+}, 2 \mathrm{C}^{-} \mathrm{d}^{-}\right)$. Oral prednisone $\left(3 \mathrm{C}_{4} \mathrm{~d}^{+}, 2 \mathrm{C} 4 \mathrm{~d}^{-}\right)$, prednisone with azathioprine $\left(5 \mathrm{C}^{+} \mathrm{d}^{+}\right.$, $\left.2 \mathrm{C}^{2} \mathrm{~d}^{-}\right)$or cyclosporine A alone $\left(1 \mathrm{C}^{2} \mathrm{~d}^{+}\right)$were given as maintenance therapy (Table 2). nIMN C4d positivity was characterized by fine granular continuous diffuse and global staining of glomerular capillary walls (Figure 1D). Tubules, interstitium and interstitial capillaries resulted always ${\mathrm{C} 4 \mathrm{~d}^{-}}^{-}$(Figure 1).

Data on 24-hour proteinuria (UPr) adjusted for $100 \mathrm{ml}$ MDRD eGFR (Modification of Diet in Renal Disease estimated Glomerular Filtration Rate) [21] and MDRD eGFR have been analysed at diagnosis, after 6 months and at latest follow-up $\left(\mathrm{C}_{4} \mathrm{~d}^{+} 31 \pm 26\right.$ months and C4d $29 \pm 31$ months $(P=\mathrm{NS})$ for $\mathrm{C}^{-} \mathrm{d}^{+}$for $\mathrm{C}^{-} \mathrm{d}^{-}$nIMNs respectively. Statistically significant higher UPr levels were observed in $\mathrm{C} \mathrm{d}^{+}$(when compared to C4d $\mathrm{d}^{-}$nIMNs at last follow-up (Table 3). Significantly lower C4d $\mathrm{d}^{+}$MDRD eGFR was found at last follow-up when compared to MDRD eGFR values at diagnosis (Table 3).

\section{Discussion}

We have described glomerular capillary C4d deposits in $66 \%$ of our nIMN kidney biopsies. C4d $\mathrm{d}^{+}$nIMNs appeared to be associated to a) a significant increase of UPr during a follow-up longer than 6 months when compared to $C 4 \mathrm{~d}^{-}$patients; b) a significant reduction of $\mathrm{C} 4 \mathrm{~d}^{+}$ MDRD eGFR has been observed at last follow-up (31 \pm 26 months) when compared to values at diagnosis. Although in experimental membranous nephropathy the role of complement for podocyte injury is essential [22] it is questioned in nIMN because the human disease is characterized mainly by $\operatorname{IgG} 4$ rather than $\operatorname{IgG} 3$ deposits [23]. It is known that IgG4 subclasses do not or weakly activate complement. Nevertheless, our data showed that $\mathrm{Cd} 4^{-}$nIMN patients had a more stable UPr and glomerular function when compared to $C 4 \mathrm{~d}^{+} \mathrm{nIMN}$ patients. Little is known on $\mathrm{C} \mathrm{d}^{+}$at a biological level, but an interesting ultrastructural study has been recently carried out on transplant glomerulopathy [24]. It revealed that $\mathrm{C}^{-} \mathrm{d}^{-}$endothelium after $\mathrm{C} 4 \mathrm{~d}$ deposition shifted from normal fenestrated to a continuous structure with expansion of mesangial matrix and late podocyte fusion. It appears consistent with our clinical finding in $\mathrm{C} 4 \mathrm{~d}^{+} \mathrm{nIMN}$ patients of a relatively slow reduction of renal function associated with an increase of UPr excretion. We are aware that our results have limitations; we know that they show the outcome of a retrospective study with a small biopsy series, and a relatively short term follow-up. Nevertheless, this 
Citation: Sepe V, Albrizio P, Canton AD (2015) C4d Glomerular Deposits and Disease Progression in Native Idiopathic Membranous Nephropathy. J Nephrol Ther 5: 212. doi:10.4172/2161-0959.1000212

Page 3 of 4

\begin{tabular}{|c|c|c|c|c|c|c|c|}
\hline \multirow{2}{*}{ Patient No. } & C4d & Age & Sex & $\mathrm{SCr}$ & eGFR & Proteinuria & nIMN \\
\hline & pos/neg & years & M/F & $\mathrm{mg} / \mathrm{dl}$ & $\mathrm{ml} / \mathrm{min}$ & g/day & stage [20] \\
\hline 1 & pos & 67 & $\mathrm{M}$ & 2.12 & 31 & 35.40 & III \\
\hline 2 & pos & 68 & $\mathrm{~F}$ & 0.94 & 59 & 6.41 & II \\
\hline 3 & pos & 24 & $F$ & 0.80 & 148 & 6.62 & 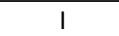 \\
\hline 4 & pos & 24 & $M$ & 0.75 & 129 & 2.16 & II \\
\hline 5 & pos & 68 & $\mathrm{~F}$ & 0.97 & 57 & 2.45 & III \\
\hline 6 & pos & 52 & $M$ & $\begin{array}{l}0.88 \\
\end{array}$ & 91 & 0.64 & 1 \\
\hline 7 & pos & 67 & $M$ & 1.20 & 60 & 10.31 & III \\
\hline 8 & pos & 17 & $\mathrm{~F}$ & 0.48 & 166 & 0.01 & II \\
\hline 9 & pos & 78 & $F$ & 1.34 & 37 & 33.88 & II \\
\hline 10 & pos & 24 & $M$ & 0.85 & 111 & 8.42 & III \\
\hline 11 & neg & 49 & $\mathrm{~F}$ & 0.88 & 66 & 1.01 & II \\
\hline 12 & neg & 39 & $M$ & 0.73 & 120 & 2.69 & III \\
\hline 13 & neg & 40 & $\mathrm{~F}$ & 1.50 & 39 & 6.11 & III \\
\hline 14 & neg & 28 & $\mathrm{~F}$ & 2.20 & 27 & 2.87 & II \\
\hline 15 & neg & 68 & $M$ & 1.90 & 35 & 10.72 & II \\
\hline
\end{tabular}

Table 1: General and laboratory findings of 15 nIMN patients tested for kidney C4d immunohistochemistry.nIMN, native Idiopathic Membranous Nephropathy; No., number; M, male; F, female; SCr, serum creatinine; eGFR, MDRD eGFR (Modification of Diet in Renal Disease estimated Glomerular Filtration Rate) [22]; proteinuria, adjusted for $100 \mathrm{ml}$ MDRD eGFR: average from 3 consecutive days sampled at the time of hospitalization; pos, C4d positive immunoperoxidase staining; neg, C4d negative immunoperoxidase staining

\begin{tabular}{|c|c|c|}
\hline Patient No. & C4d (pos/neg) & Induction \\
\hline 1 & pos & oral prednisone $1 \mathrm{mg} / \mathrm{Kg} /$ day for 1 month \\
\hline 2 & pos & i.v. methylprednisolone $1 \mathrm{~g}$ for 3 consecutive days \\
\hline 3 & pos & i.v. methylprednisolone $1 \mathrm{~g}$ for 3 consecutive days \\
\hline 4 & pos & i.v. methylprednisolone $1 \mathrm{~g}$ for 3 consecutive days \\
\hline 5 & pos & i.v. cyclophosphamide $1 \mathrm{~g} /$ month for 6 months \\
\hline 6 & pos & i.v. methylprednisolone $1 \mathrm{~g}$ for 3 consecutive days \\
\hline 7 & pos & i.v. cyclophosphamide $1 \mathrm{~g} /$ month for 6 months \\
\hline 8 & pos & i.v. methylprednisolone $1 \mathrm{~g}$ for 3 consecutive days \\
\hline 9 & pos & i.v. cyclophosphamide $1 \mathrm{~g} /$ month for 6 months \\
\hline 10 & pos & i.v. cyclophosphamide $1 \mathrm{~g} /$ month for 6 months \\
\hline 11 & neg & \\
\hline 13 & neg & i.v. cyclophosphamide $1 \mathrm{~g} /$ month for 6 months \\
\hline 14 & neg & i.v. cyclophosphamide $1 \mathrm{~g} /$ month for 6 months \\
\hline 15 & neg & i.v. methylprednisolone $1 \mathrm{~g}$ for 3 consecutive days \\
\hline & neg & i.v. methylprednisolone $1 \mathrm{~g}$ for 3 consecutive days \\
\hline
\end{tabular}

\begin{tabular}{|c|}
\hline Maintenance \\
\hline prednisone $5 \mathrm{mg} /$ day \\
\hline prednisone $10 \mathrm{mg} / \mathrm{day}$ \\
\hline prednisone $10 \mathrm{mg} /$ day \\
\hline prednisone $5 \mathrm{mg} /$ day + cyclosporine $300 \mathrm{mg} /$ day \\
\hline prednisone $5 \mathrm{mg} /$ day + azathioprine $50 \mathrm{mg} /$ day \\
\hline prednisone $5 \mathrm{mg} /$ day + azathioprine $50 \mathrm{mg} /$ day \\
\hline prednisone $12.5 \mathrm{mg} /$ day + azathioprine $100 \mathrm{mg} /$ day \\
\hline prednisone $5 \mathrm{mg} /$ day \\
\hline prednisone $12.5 \mathrm{mg} /$ day + azathioprine $100 \mathrm{mg} /$ day \\
\hline cyclosporine $125 \mathrm{mg} /$ day \\
\hline none \\
\hline prednisone $12.5 \mathrm{mg} /$ day + azathioprine $100 \mathrm{mg} /$ day \\
\hline prednisone $12.5 \mathrm{mg} /$ day + azathioprine $100 \mathrm{mg} /$ day \\
\hline prednisone $5 \mathrm{mg} / \mathrm{day}$ \\
\hline prednisone $10 \mathrm{mg} /$ day \\
\hline
\end{tabular}

Table 2: Treatment details of 15 nIMN patients tested for C4d immunohistochemistry on renal biopsy. nIMN, native Idiopathic Membranous Nephropathy; No., number; pos, C4d positive immunohistochemistry; neg, C4d negative immunohistochemistry; induction, induction therapy at diagnosis; maintenance, maintenance therapy during follow-up.

\begin{tabular}{|c|c|c|c|c|}
\hline \multicolumn{2}{|c|}{ nIMN patients } & \multicolumn{3}{|c|}{ Proteinuria, g/day; $M \pm S D$} \\
\hline & No & At diagnosis & 6-month follow-up & Last follow-up \\
\hline C4d positive & 10 & $10.63 \pm 13.10$ & $12.98 \pm 24.48$ & $4.75 \pm 3.10^{*}$ \\
\hline C4d negative & 5 & $4.68 \pm 3.85$ & $3.87 \pm 3.60$ & $1.34 \pm 1.15$ \\
\hline \multicolumn{2}{|c|}{ nIMN patients } & \multicolumn{3}{|c|}{ MDRD eGFR, ml/min; mean \pm standard deviation } \\
\hline & No & At diagnosis & 6-month follow-up & Last follow-up \\
\hline C4d positive & 10 & $88.9 \pm 47.5$ & $74.5 \pm 52.4$ & $56.20 \pm 28.91^{* *}$ \\
\hline C4d negative & 5 & $57.4 \pm 37.9$ & $71.0 \pm 48.6$ & $43.7 \pm 41.0$ \\
\hline
\end{tabular}

Table 3: Proteinuria and renal function in ten C4d negative and five C4d positive nIMN patients.Proteinuria, daily proteinuria adjusted for 100 mI MDRD eGFR (Modification of Diet in Renal Disease estimated Glomerular Filtration Rate) [22]; ${ }^{*} P=0.0051$ vs. C4d positive nIMN patients at last follow-up; MDRD eGFR, ${ }^{* *} P=0.0337$ vs. C4d positive at diagnosis.

is not the first time that $\mathrm{C} 4 \mathrm{~d}$ renal deposits are associated with negative disease progression. As previously described for IgAN [6] also for nIMN we have revealed that $\mathrm{C} 4 \mathrm{~d}$ immunohistochemistry is a valuable marker of disease worsening. In our opinion the demonstration of glomerular capillary wall C4d deposits might justify a more aggressive immunosuppressive regimen as already standardized in clinical transplantation. It is auspicable that larger nIMN series with longer follow-up would confirm our finding that glomerular $\mathrm{C}_{4} \mathrm{~d}^{+}$is a reliable clinical predictive marker of nIMN progression.

\section{References}

1. Sheerin NS, Risley P, Abe K, Tang Z, Wong W, et al. (2008) Synthesis of 
Citation: Sepe V, Albrizio P, Canton AD (2015) C4d Glomerular Deposits and Disease Progression in Native Idiopathic Membranous Nephropathy. J Nephrol Ther 5: 212. doi:10.4172/2161-0959.1000212

Page 4 of 4

complement protein C3 in the kidney is an important mediator of local tissue injury. FASEB J 22: 1065-1072.

2. Colvin RB (2009) Pathology of chronic humoral rejection. Contrib Nephrol 162 75-86.

3. Einecke G, Sis B, Reeve J, Mengel M, Campbell PM, et al. (2009) Antibodymediated microcirculation injury is the major cause of late kidney transplant failure. AM J Transplant 9: 2520-2531.

4. Murata K, Baldwin WM $3^{\text {rd }}$ (2009) Mechanisms of complement activation, C4d deposition, and their contribution to the pathogenesis of antibody-mediated rejection. Transplant Rev (Orlando) 23:139-150.

5. Zwirner J, Felber E, Herzog V, Riethmüller G, Feucht HE (1989) Classical pathway of complement activation in normal and diseased human glomeruli. Kidney Int 36: 1069-1077.

6. Espinosa M, Ortega R, Gómez-Carrasco JM, López-Rubio F, López-Andreu $\mathrm{M}$, et al. (2009) Mesangial C4d deposition: a new prognostic factor in IgA nephropathy. Nephrol Dial Transplant 24:886-891.

7. Oortwijn BD, Rastaldi MP, Roos A, Mattinzoli D, Daha MR, et al. (2007) Demonstration of secretory $\lg A$ in kidneys of patients with $\lg A$ nephropathy. Nephrol Dial Transplant 22: 3191-3195.

8. Roos A, Rastaldi MP, Calvaresi N, Oortwijn BD, Schlagwein N, et al. (2006) Glomerular activation of the lectin pathway of complement in IgA nephropathy is associated with more severe renal disease. J Am Soc Nephrol 17: 1724-1734.

9. Xing GQ, Chen M, Liu G, Zheng X, E J, Zhao MH (2010) Differential deposition of $\mathrm{C} 4 \mathrm{~d}$ and $\mathrm{MBL}$ in glomeruli of patients with ANCA-negative pauci-immune crescentic glomerulonephritis. J Clin Immunol 30: 144-156.

10. Manoharan A, Madaio MP (2010) Biomarkers in lupus nephritis. Rheum Dis Clin North Am 36: 131-143.

11. Zwirner J, Felber E, Burger R, Bitter-Suermann D, Riethmüller G, et al (1993) Classical pathway of complement activation in mammalian kidneys. Immunology 80: 162-167.

12. Cohen D, Koopmans M, Kremer Hovinga IC, Berger SP, Roos van Groningen $\mathrm{M}$, et al. (2008) Potential for glomerular C4d as an indicator of thrombotic microangiopathy in lupus nephritis. Arthritis Rheum 58: 2460-2469.

13. Li SJ, Liu ZH, Zen CH, Wang QW, Wang Y, et al. (2007) Peritubular capillary C4d deposition in lupus nephritis different from antibody-mediated renal rejection. Lupus 16: 875-880.

14. Beck LH Jr, Bonegio RG, Lambeau G, Beck DM, Powell DW, et al. (2009) M-type phospholipase $\mathrm{A} 2$ receptor as target antigen in idiopathic membranous nephropathy. N Engl J Med 361: 11-21.

15. Ponticelli C (2007) Membranous nephropathy. J Nephrol 20: 268-287.

16. Moulds JM (2009) Introduction to antibodies and complement. Transfus Apher Sci 40: 185-188.

17. Ranjan P, Nada R, Jha V, Sakhuja V, Joshi K (2008) The role of C4d immunostaining in the evaluation of the causes of renal allograft dysfunction. Nephrol Dial Transplant 23: 1735-1741.

18. Solez K, Colvin RB, Racusen LC, Haas M, Sis B, et al. (2008) Banff 07 classification of renal allograft pathology: updates and future directions. Am J Transplant 8: 753-760.

19. Schwartz MM (1998) Membranous glomerulonephritis. In: Heptinstall's pathology of the kidney ( $5^{\text {th }}$ edn), JC Jennette, JL Olson, MM Schwartz and FG Silva (edt), Lippincott-Raven Publischers, Philadelphia.

20. Choi J, Cho YM, Yang WS, Park TJ, Chang JW, et al. (2007) Peritubular capillary C4d deposition and renal outcome in post-transplant IgA nephropathy. Clin Transplant 21: 159-165.

21. http://www.sin-italy.org/Governo_Clinico/calcolatori_clinici.asp.

22. Salant DJ, Belok S, Madaio MP, Couser WG (1980) A new role for complement in experimental membranous nephropathy in rats. J Clin Invest 66: 1339-1350.

23. Haas M (1994) IgG subclass deposits in glomeruli of lupus and nonlupus membranous nephropathies. Am J Kidney Dis. 23: 358-364.

24. Wavamunno MD, O'Connell PJ, Vitalone M, Fung CL, Allen RD, et al. (2007) Transplant glomerulopathy: ultrastructural abnormalities occur early in longitudinal analysis of protocol biopsies. Am J Transplant 7: 2757-2768. 